\title{
Optimising sulfuric acid hard coat anodising for an Al-Mg-Si wrought aluminium alloy*
}

\author{
N. BARTOLO ${ }^{1}$, E. SINAGRA $^{1}$, B. MALlia $^{2 \dagger}$ \\ ${ }^{1}$ Department of Chemistry, University of Malta, Msida, MSD2080, Malta \\ ${ }^{2}$ Department of Metallurgy and Materials Engineering, University of Malta, Msida, MSD2080, Malta
}

\begin{abstract}
This research evaluates the effects of sulfuric acid hard coat anodising parameters, such as acid concentration, electrolyte temperature, current density and time, on the hardness and thickness of the resultant anodised layers. A small scale anodising facility was designed and set up to enable experimental investigation of the anodising parameters. An experimental design using the Taguchi method to optimise the parameters within an established operating window was performed. Qualitative and quantitative methods of characterisation of the resultant anodised layers were carried out. The anodised layer's thickness, and morphology were determined using a light optical microscope (LOM) and field emission gun scanning electron microscope (FEG-SEM). Hardness measurements were carried out using a nano hardness tester. Correlations between the various anodising parameters and their effect on the hardness and thickness of the anodised layers were established. Careful evaluation of these effects enabled optimum parameters to be determined using the Taguchi method, which were verified experimentally. Anodised layers having hardness varying between $2.4-5.2 \mathrm{GPa}$ and a thickness of between $20-80 \mu \mathrm{m}$ were produced. The Taguchi method was shown to be applicable to anodising. This finding could facilitate on-going and future research and development of anodising, which is attracting remarkable academic and industrial interest.
\end{abstract}

Keywords: aluminium; hard coat anodizing; sulfuric acid; Taguchi method; nano pores; nano hardness

(C) Wroclaw University of Technology.

\section{Introduction}

More stringent environmental legislation requires a reduction in fuel consumption and less carbon emissions. This has made the use of light alloys more attractive [1-3]. Aluminium alloys display high strength to weight ratio, natural abundance and competitive cost compared to other materials. This is driving their increased use in engineering applications.

The main challenges with the use of aluminium alloys include joining and surface treatment issues [3]. Major surface related problems of aluminium and its alloys are their generally poor tribological performance, corrosion response and the poor adhesion of protective coatings. Aluminium alloys are generally not good candidate materials

\footnotetext{
${ }^{*}$ This paper was presented at the $9^{t h}$ Conference on Functional and Nanostructured Materials, FNMA'12, Aegina Island (Greece) 2012.

†E-mail: Bertram.mallia@um.edu.mt
}

for applications involving abrasive wear as a result of their low hardness $[4,5]$. They are vulnerable to corrosion, since the spontaneously formed oxide layer does not offer adequate corrosion resistance [4]. This excludes unprotected aluminium from demanding applications, necessitating specialised protection of aluminium [6].

A proven chemical surface engineering technology to increase corrosion and wear resistance is anodising. This is a chemical surface engineering technique that oxidises and converts the surface of aluminium to a uniform and continuous alumina oxide film [7]. Alumina has a relatively high hardness (2.9 - 5.9 GPa) and is chemically inert $[4,6,8]$.

The properties of the anodised layer are dependent on its thickness, the composition of the underlying substrate material and the anodising conditions: electrolyte temperature, voltage, current density and anodising time $[4,9,10]$.

The aims of this research were to select and optimise a combination of hardness and thickness of 
the sulfuric acid hard coat anodised layers on an aluminium silicon magnesium wrought aluminium alloy. This was achieved by investigating the interdependent effects of the various anodising parameters which were the acid concentration, electrolyte temperature, current density and anodising time. These parameters were investigated using the Taguchi method, which allows the variation of more than one parameter per experiment, therefore narrowing down the number of experiments whilst still giving a good representation of the independent effects of each anodising parameter.

In open literature, there is a lack of information on the combined and sometimes opposing effects of anodising parameters on the hardness and thickness of anodised hard coats. In this work the anodising parameters of sulfuric acid hard coat anodising are investigated systematically to determine their effects on the anodised layer characteristics. This is important given that chromic acid anodising is harmful to the environment and should be replaced by alternative processes [7].

\section{Materials and methods}

\subsection{Substrate material}

The substrate material used was an Al98.81 wt.\% Mg-0.92 wt.\% Si-0.26 wt.\% six series wrought alloy flat bar as determined using EDAX. The flat bar with a thickness of $0.2 \mathrm{~cm}$ was cut to produce $9 \times 2 \mathrm{~cm}$ rectangular specimens. Two sequential cleaning procedures were carried out to ensure that both organic (mainly machining lubricants) and inorganic (mainly thin oxide films) contaminants were removed from the surface of the specimens. Inorganic soil was removed by dipping the specimen in a solution of $120 \mathrm{~g} / \mathrm{L}$ ammonium biflouride (dissolved in $9.5 \% \mathrm{v} / \mathrm{v}$ nitric acid), whilst organic soil was removed using acetone. This produced a metallurgically clean surface which could be easily replicated in industry.

\subsection{Experimental setup}

A schematic of the anodising facility used in this work is shown in Fig. 1. The anodising facility permitted the control of the anodising param- eters independently. These were the acid temperature, acid concentration, current density and anodising time.

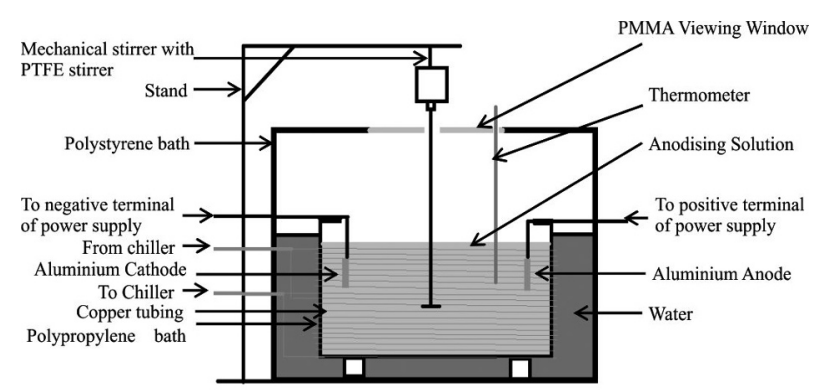

Fig. 1. Schematic diagram of the anodising bath.

The thermostatically controlled anodising bath consisted of a polypropylene rectangular bath. Copper tubing was wound around the anodising bath connected to a thermostatically controlled chiller. The polypropylene bath was placed inside a larger sealed polystyrene insulating unit and the space between them was filed with water contained in the larger insulating bath (Fig. 1).

Chilled coolant was circulated through the copper tubing to cool down the anodising solution to the set temperature. The water contained in the polystyrene bath increased the cooling effectiveness and acted as a buffer to maintain constant anodising solution temperature.

A mechanical stirrer was used to stir the anodising solution and ensure a uniform electrolyte temperature throughout. This was effective to dissipate quickly the heat evolved at the specimenelectrolyte interface during anodising.

A Delta Elektronica power supply (SM7020-D) was used to enable anodising at constant current. Since the anodised layer being formed was insulative in nature, electrical resistance increased with the anodised layer thickness. The power supply automatically compensated for the increased resistance by an increase in voltage to maintain the set anodising current.

\subsection{Anodising process parameters}

The process parameters were selected to incorporate the entire range used for sulfuric acid hardcoat anodising. The parameter ranges used in 
this study were verified to be appropriate to the process through preliminary experimentation and were set as follows: current density between 1.36 $-2.26 \mathrm{~A} / \mathrm{dm}^{2}$, sulfuric acid concentration between $165.3-330.6 \mathrm{~g} / \mathrm{L}$, temperature between $0-20{ }^{\circ} \mathrm{C}$ and anodising time between 30 - 90 minutes.

Table 1 shows the experimental parameter settings (orthogonal array) used in the Taguchi experiment. Each experiment was repeated three times.

\subsection{Anodising procedure}

A pre-cleaned specimen was connected to the anodic terminal jig and immersed in solution. The mechanical stirrer was then switched on at a rotational speed of $180 \mathrm{rpm}$. This resulted in stirring of the anodising solution with minimal agitation. The power supply and stop watch were then switched on simultaneously. Two minutes were allowed for the voltage to stabilize which was considered to be the initial anodising voltage. The temperature was monitored throughout the experiment at 10 minute intervals in order to ensure that no temperature fluctuations occur.

The specimen was then removed from solution and cleaned. Cleaning was performed by first immersing the specimen in deionized water for 30 seconds followed by a 10 second dip in $9.5 \%$ $\mathrm{v} / \mathrm{v}$ nitric acid and finally a 30 second dip in deionized water. The dipping procedures involved stirring of the specimen in the solution to ensure maximum dissolution of the surface contaminants. The specimen was then wiped with tissue paper, dried using compressed air for a few seconds and was then stored in a sealed plastic bag.

\subsection{Structure, thickness and topography of the anodised layers}

The structure of the anodised layers was investigated using a Bruker D8 X-Ray Diffractometer (XRD) operated in locked couple mode and using Mo-K $\alpha$ radiation. The thickness of the anodised layers were determined on polished cross sections of the specimen using a Nikon Optophot100, light optical microscope, (LOM) at 500x magnification. The topography of the anodised layers was studied using field emission gun scanning electron microscope (FEG-SEM) secondary electron images obtained using an Ultra Plus HR Carl Zeiss NTS FEG-SEM at 1,000, 10,000 and 35,000x magnification respectively (Fig. $5-7$ ). The specimens were coated with a thin layer of graphite using an Agar Autocarbon coater to mitigate surface charging.

\subsubsection{Nano hardness testing}

A nano hardness tester (micro materials nano test 600) was used to measure surface hardness. For these measurements, the experimental parameters consisted of a loading/unloading rate of $1.5 \mathrm{mN} / \mathrm{s}$ and a dwell time at maximum load of $30 \mathrm{~s}$. These parameters ensured that the indenter never penetrated more than $10 \%$ of the anodised layer thickness (maximum indentation distance was set to $900 \mathrm{~nm}$ ). This eliminated substrate effects in the nano hardness measurement. For each specimen 20 indents were performed ( 5 columns with 4 indents per column). The distance between each column and row was set to $50 \mu \mathrm{m}$. A tailor made jig was designed to be able to mount all nine Taguchi specimens together (in two rows). This enabled the testing of all the specimens in one schedule, resulting in a more robust experiment.

\section{Results}

\subsection{Structure, thickness and topography of the anodised layers}

The diffractograph of the anodised layer showed a broad amorphous peak, and superimposed on it were the characteristic aluminium alloy FCC peaks. No additional peaks from the anodised layer were observed.

The anodised layer thickness varied substantially $(17-87 \mu \mathrm{m})$ between all the Taguchi experiments (Fig. 2). There was no abnormal micro porosity or surface roughness observed using the LOM at 500× magnification (Fig. 3). The difference in the measured layer thickness of the 3 repeated experiments for each Taguchi set was minimal, typically in the range of less than $5 \%$ of the total anodised layer thickness. (Fig. 2) A Student $t$-test showed that the thickness of the various 
Table 1. Taguchi Parameter Orthogonal Array.

\begin{tabular}{ccccc}
\hline $\begin{array}{c}\text { Experiment } \\
\text { number }\end{array}$ & $\begin{array}{c}\text { Concentration } \\
(\mathrm{g} / \mathrm{L})\end{array}$ & $\begin{array}{c}\text { Temperature } \\
\left({ }^{\circ} \mathrm{C}\right)\end{array}$ & $\begin{array}{c}\text { Current density } \\
\left(\mathrm{A} / \mathrm{dm}^{2}\right)\end{array}$ & $\begin{array}{c}\text { Time } \\
(\mathrm{min})\end{array}$ \\
\hline \hline 1 & 165.3 & 0 & 1.36 & 30 \\
2 & 165.3 & 10 & 1.81 & 60 \\
3 & 165.3 & 20 & 2.26 & 90 \\
4 & 247.9 & 0 & 1.81 & 90 \\
5 & 247.9 & 10 & 2.26 & 30 \\
6 & 247.9 & 20 & 1.36 & 60 \\
7 & 330.6 & 0 & 2.26 & 60 \\
8 & 330.6 & 10 & 1.36 & 90 \\
9 & 330.6 & 20 & 1.81 & 30 \\
\hline
\end{tabular}

* Temperature was monitored during anodising every 10 minutes with an accuracy of $0.5^{\circ} \mathrm{C}$.

anodised layers were statistically significant with $95 \%$ confidence level.

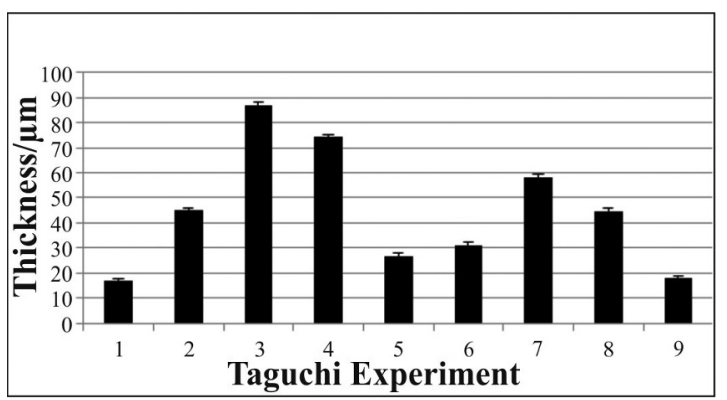

Fig. 2. Mean anodised layer thickness for the different Taguchi experiments. Error bars represent $\pm 1 \theta$.

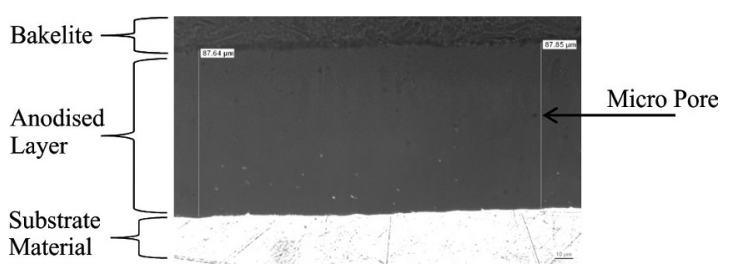

Fig. 3. A cross section LOM image of polished specimen $3 \mathrm{~B}$ at 500x magnification.

The Taguchi experimental data was analysed using ANOVA. This determined the influence of the anodising parameters on the anodised layer thickness. The resultant correlations of the individual parameters on layer thickness are shown in Fig. 4.

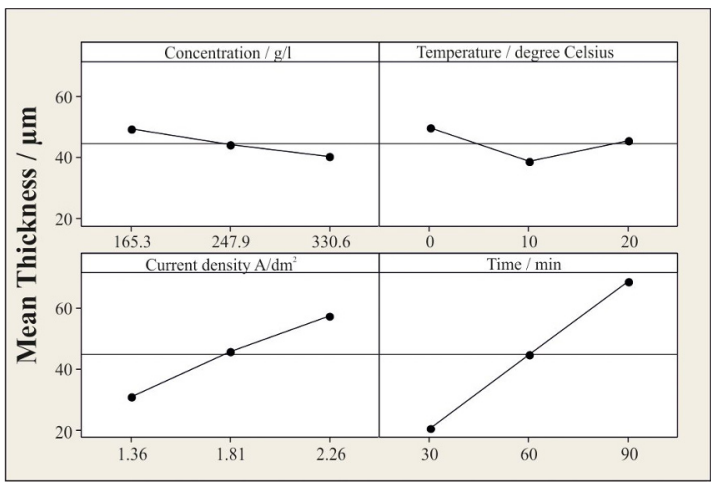

Fig. 4. Main effects plots for mean anodised layer thickness changes with solution concentration, solution temperature, current density and anodising time.

Electrographs taken by a FEG-SEM show general and detailed topographical features at 1,000 $\times$ (Fig. 5) and 10,000 $\times$ (Fig. 6) magnification respectively. Nanostructural features within the layers were viewed at 35,000 x magnification (Fig. 7).

\subsection{Nano hardness results}

Twenty nano indentations were carried out for each Taguchi experiment. Their corresponding load versus displacement graphs were plotted (Fig. 8). The average nano hardness for each set of experiments was calculated using the integrated nanoindentation software and plotted (Fig. 9). Experi- 


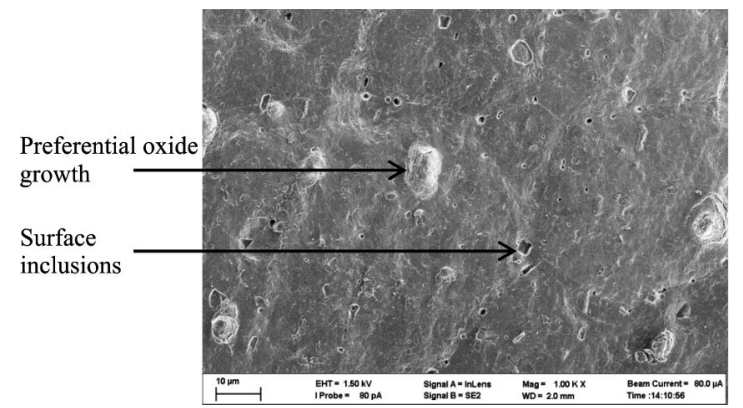

Fig. 5. Secondary electron topographical FEGSEM image of specimen 1 showing general morphology.

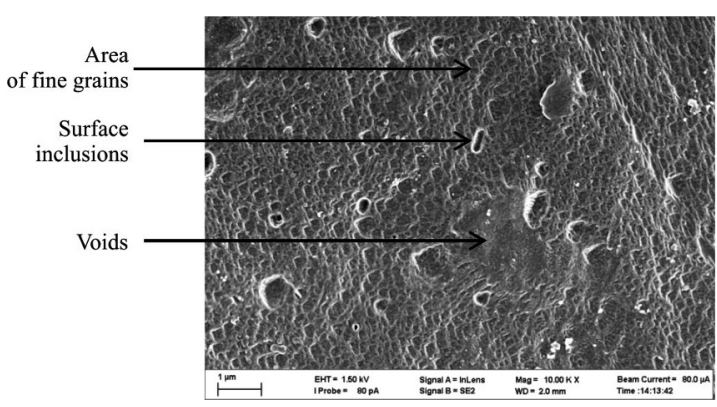

Fig. 6. Secondary electron topographical FEGSEM image of specimen 1 showing detailed morphology.

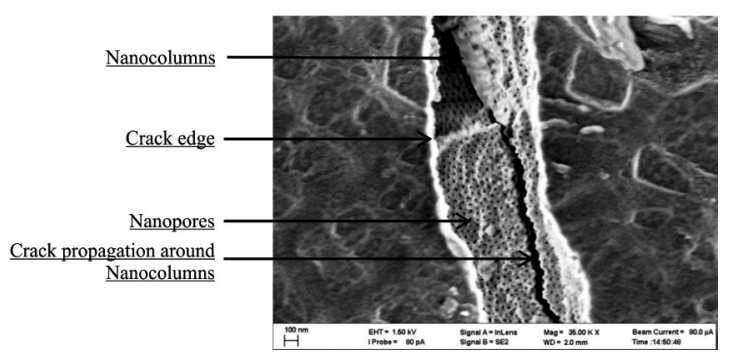

Fig. 7. Secondary electron topographical FEG-SEM image of a specimen 4 showing ordered nanopores and their respective columnar morphology.

ments $1,2,4,5,6,7,8$ and 9, were significantly harder than experiment 3 which was carried out at the highest anodising temperature, current density and time.

The Taguchi experimental hardness data was analysed using one-way ANOVA. This determined the influence of the anodising parameters on the anodised layer nano hardness. The resultant correla-

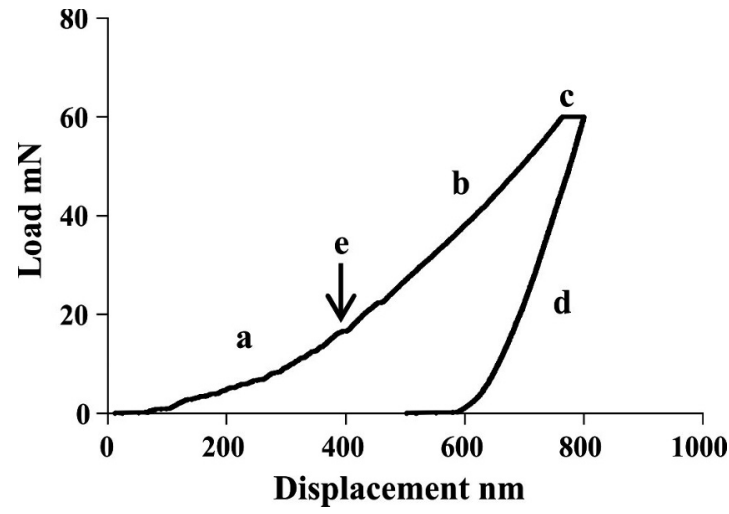

Fig. 8. Representative graph of load $(\mathrm{mN})$ vs. displacement (nm); (a) curved loading part, (b) straight loading part, (c) creep at constant maximum load, (d) unloading part and (e) pop-in phenomenon.

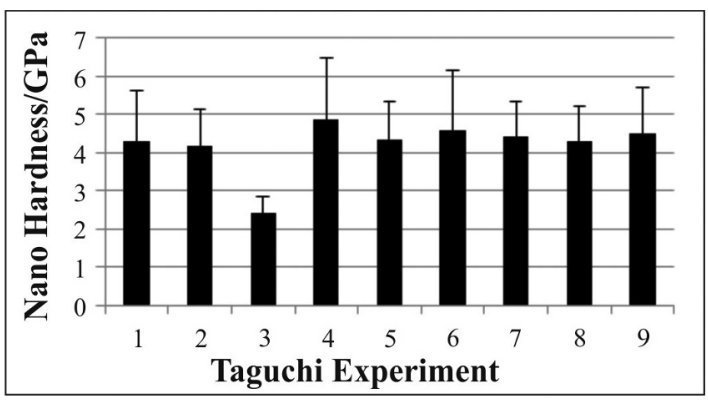

Fig. 9. Mean anodised layer nano hardness for the different Taguchi experiments. Error bars represent $\pm 1 \theta$.

tions of the individual parameters on nano hardness are shown in Fig. 10.

\section{Discussion}

In agreement with literature [4], the anodised layers produced in this study were amorphous (Fig. 11). The Taguchi experiment reproduced the entire known sulfuric acid hard coat range of $2.9-$ $5.9 \mathrm{GPa}$ [4] (Fig. 9). The lowest hardness attained for the anodised layer was $2.4 \mathrm{GPa}$, whilst the highest hardness measured was $4.9 \mathrm{GPa}$. The hardness of the remainder of the Taguchi sets were close to $4 \mathrm{GPa}$. Variance between the repeated load versus displacement graphs on the same specimen (Fig. 9) are thought to be the result of the different indent- 


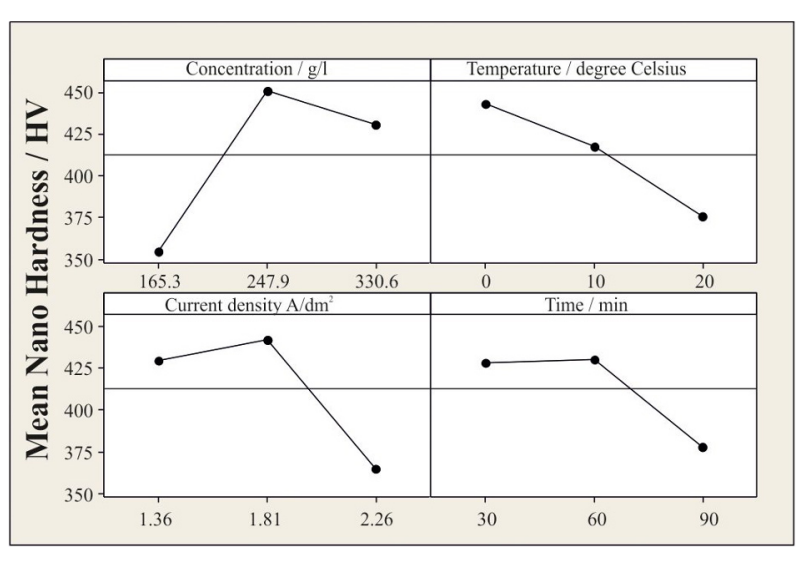

Fig. 10. Main effects plots for mean nano hardness changes with solution concentration, solution temperature, current density and anodising time.

ing angles with respect to the columnar structure of the anodised layer. The difference in column angles with respect to the sample surface is a result of surface roughness on the substrate material (mainly due to the etching process) being replicated in the anodised layer. This occurs as the columnar structure orients itself perpendicular to the substrate material [11].

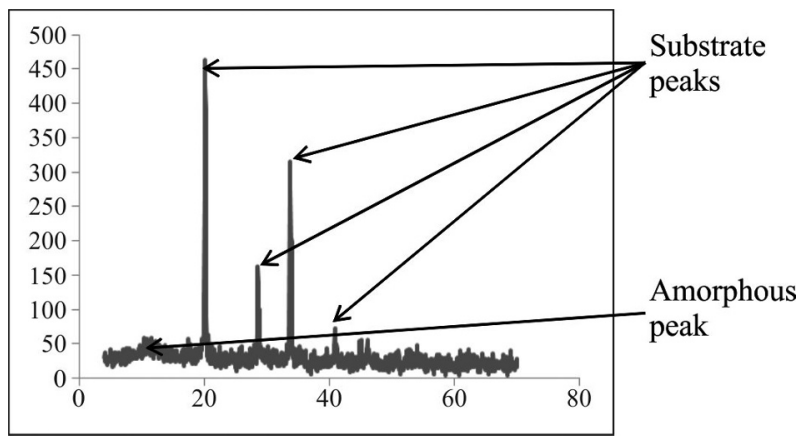

Fig. 11. XRD pattern for substrate and anodised substrate.

Varying sulfuric acid concentration had a relatively small effect on anodised layer thickness in comparison with current density and anodising time. A small decrease in layer thickness was observed with increased acid concentration (Fig. 4). It has been suggested that higher concentrations of acid aids the dissolution process resulting in thinner oxide films [11]. The small decrease in thick- ness with increasing acid concentration suggests that the rate of oxide formation is largely governed by the anodising current density and not by the concentration of the oxidising agent $\left(\mathrm{SO}_{4}^{2-}\right)$ present, when the latter is in excess. Excessive concentration of acid favours oxide dissolution resulting in thinner anodised layers. Acid concentration was observed to have two opposing effects on hardness. For the low to medium concentrations range (165.3 to $247.9 \mathrm{~g} / \mathrm{L}$ sulfuric acid) an increase in hardness was observed whilst a decrease in hardness resulted for the medium to high concentrations (247.9 to $330.6 \mathrm{~g} / \mathrm{L}$ sulfuric acid) (Fig. 10). It has been reported that the morphology of the anodised layer has an influence on the mechanical properties. In particular, thicker column walls give the coating increased load bearing capacity, hence increased measured hardness [8]. A possible explanation for the observed initial increase in hardness with an increasing acid concentration is the formation of a denser oxide layer as a result of the oxidising agent $\left(\mathrm{SO}_{4}^{2-}\right)$ being more abundant. Dissolution is pronounced towards the surface of the oxide, as it is exposed to the solution for the longer time. This is known as pore mouth widening which results in cone-like columns [11]. This leads to a decrease in surface hardness. The initial decrease in surface hardness of the anodised layer was evident during nano-hardness testing with the loaddisplacement graphs displaying a shallow gradient towards the start of the indentation cycle (part (a) in Fig. 8). Similar graphs were also reported by TeHua [12] in recent nano indentation studies on anodised aluminium. This phenomenon is suspected to be the cause of the decrease in hardness when the acid concentration was increased further (247.9 to $330.6 \mathrm{~g} / \mathrm{L}$ sulfuric acid) since the oxidising agent is in excess, and therefore does not have a substantial effect on the oxidation reaction. On the other hand, the increased concentration of $\mathrm{H}_{3} \mathrm{O}^{+}$increases the rate of dissolution, producing a weaker anodised layer especially at the surface due to extensive pore mouth widening.

The acid temperature range $\left(0\right.$ to $\left.20{ }^{\circ} \mathrm{C}\right)$ had a smaller effect on anodised layer thickness compared to current density and anodising time. It can be presumed that this temperature range did not af- 
fect the dynamic equilibrium state of the anodising chemical process. On the other hand, increasing temperature of the anodising solution $\left(0-20{ }^{\circ} \mathrm{C}\right)$ substantially lowered the hardness of the oxide layers (Fig. 10). The kinetics for the reaction of the oxidising agent $\left(\mathrm{SO}_{4}^{2-}\right)$ are dictated by the electrostatic attraction towards the anodic specimen surface and therefore temperature plays a minor role. The $\mathrm{H}_{3} \mathrm{O}^{+}$species (the agent promoting dissolution) is repelled from the anodic specimen surface thus the dissolution process is likely to be influenced by temperature. The net effect is a noticeable increase in the rate of dissolution at the surface with increasing acid temperature, promoting pore mouth widening [13].

Anodising current density had a large effect on thickness. In agreement with literature, an increase in current density increased oxide anodised layer thickness [4]. Current density should be considered as a reagent in anodic oxidation since its increase will enhance the rate of reaction. Increasing the current density should increase the rate of sulfate ion bombardment on the anodic specimen, resulting in a higher rate of oxide formation. According to Runge and Pomis [14], an increase in current density is reported to have an effect of decrease of nucleation sites, leading to a microstructure having fewer but wider column walls (decreasing total pore volume). The thicker columns are capable of withstanding higher forces before fracture [8]. Higher current densities lead to the oxidising agent $\left(\mathrm{SO}_{4}^{2-}\right)$ arriving at the substrate surface at an increased kinetic rate, which results in a faster overall chemical oxidation reaction. This will limit the number of nucleation sites formed as a result of faster columnar growth. In accordance to this, an increase in hardness was observed with an increase in current density between 1.18 to $1.36 \mathrm{~A} / \mathrm{dm}^{2}$ (Fig. 10). However, it was observed that a further increase in current density $\left(1.18\right.$ to $\left.2.26 \mathrm{~A} / \mathrm{dm}^{2}\right)$ resulted in a decrease in hardness (Fig. 10) which was not reported by Runge and Pomis, but this could be a result of the current density parameter window not being the same. This cannot be verified since the current densities used were not stated.

Increasing anodising time increases oxide film thickness, although eventually a specific thickness is reached, where, at that point, the rate of oxide formation and dissolution are in equilibrium [11, 15]. Fig. 4. shows that the layer thickness increased linearly with time. This implies that in the Taguchi experiments the limiting thickness was not reached. Variation of anodising time between 30 to 60 minutes did not affect the anodised layer hardness (Fig. 10). This shows that dissolution during this time period was not significant. Hardness decreased when anodising was carried out for longer than 60 minutes (Fig. 10). A plausible explanation to this is that since the increased rate of dissolution is an effect of surface area of the pores, as anodising time increases the dissolution rate of the oxide increases leading to pore mouth widening and hence a weaker anodised layer.

The optimum target hard coat was the hardest anodised layer at the best possible thickness. This was attained using the Taguchi predictive model. It is clear from the results obtained that all four parameters investigated affect, although to varying degrees, both hardness and thickness of the anodised layers. Since one parameter might affect both investigated layer characteristics, careful manipulation of the parameter settings should be carried out to produce the required anodised layer. The effects of the anodising parameters on hardness and thickness should not to be viewed in isolation of other important factors. Surface roughness and risk of cracking of the anodised layer all have to be taken into consideration as they would have an effect on the mechanical integrity and tribological behaviour of the anodised layers.

It is suggested that sulfuric acid concentration is set high enough to aid efficient and favourable structural oxide formation (thick column walls), but not too high that it will have an effect of dissolving the outermost pores which result in an inferior oxide and decrease anodising efficiency. From this study the optimised concentration of sulfuric acid was $247.9 \mathrm{~g} / \mathrm{L}$.

The relation between temperature and hardness was significant. A lower temperature produced a harder anodised layer. For an anodised layer of maximum hardness an anodising temperature of $0{ }^{\circ} \mathrm{C}$ is optimal. However, on an industrial scale, moderately hard anodised layers of around $4.2 \mathrm{GPa}$ 
were possible to produce (Experiment 5 - Fig. 9) at $10{ }^{\circ} \mathrm{C}$ and may be considered for economic reasons.

The optimum current density was $1.36 \mathrm{~A} / \mathrm{dm}^{2}$. This gave the highest anodised layer surface hardness. Higher currents were observed to increase the likelihood of cracks forming without any real increase in hardness, resulting in a less robust oxide film.

Anodising time is a critical parameter, since it not only governs the attainable film thickness but also affects the mechanical properties of the outermost oxide. Hardness was not seen to be influenced in the 30-60 minutes anodising range. However, the anodised layer thickness increased linearly. During the $60-90$ minutes anodising duration the oxide experienced a reduction in hardness, whilst the oxide layer continued to gain thickness. This implies that in order to have a thick anodised layer with optimum hardness, anodising to a maximum of 60 minutes would be sufficient to produce a layer of approximately $50 \mu \mathrm{m}$.

These optimum parameters (acid concentration $247.9 \mathrm{~g} / \mathrm{L}$, temperature of $0{ }^{\circ} \mathrm{C}$, current density of $1.81 \mathrm{~A} / \mathrm{dm}^{2}$, anodising time of 60 minutes) which were obtained by using the Taguchi model predicted an optimum hardness of 5.2 GPa and a thickness of $48 \mu \mathrm{m}$. The predicted layer characteristics were verified experimentally and produced a sound crack free anodised layer with a hardness of 5.2 GPa and a thickness of $48 \mu \mathrm{m}$, showing the applicability of this experimental design.

\section{Conclusion}

Sulfuric acid proved to be an environmentally acceptable anodising electrolyte, which produced the required anodising hard coat range: thickness $30-50 \mu \mathrm{m}$ and hardness $2.4-5.2 \mathrm{GPa}$, similar to that produced using chromic acid which is not environmentally acceptable.

Hard coat anodising resulted in an increase between 5 and 7 fold in hardness of the Al98.81 wt.\% Mg-0.92 wt.\% Si-0.26 wt.\% extruded aluminium alloy.

The results obtained demonstrated well the interdependent effects of the various anodising pa- rameters (acid concentration, temperature, current density and anodising time) on hardness and thickness of the anodised layer. Both current density and anodising time had a strong effect on film thickness, whereas acid concentration and anodising temperature had relatively lower effects on film thickness. It was also established that all the anodising parameters had major effects on the anodised layer hardness.

The optimised parameters for hard coat anodising of $\mathrm{Al}-98.81$ wt.\% $\mathrm{Mg}-0.92$ wt.\% Si-0.26 wt.\% aluminium extrusion were established using the Taguchi predictive model at acid concentration of $247.9 \mathrm{~g} / \mathrm{L}$, temperature of $0{ }^{\circ} \mathrm{C}$, current density of $1.81 \mathrm{~A} / \mathrm{dm}^{2}$ and anodising time of 60 minutes. The predictive optimal hardness and thickness were in good agreement with the values obtained experimentally. These were a hard coat hardness of $5.16 \mathrm{GPa}$ and a thickness of $48 \mu \mathrm{m}$. Thus, the Taguchi method proved to be applicable to anodising investigation and process optimisation. It is a powerful tool to aid R\&D of anodic anodising processes, which at present is of great academic and industrial interest.

\section{Acknowledgements}

The authors are indebted to funding through ERDF 012 (Malta) for the financing of the testing and characterisation equipment through the project: "Developing an Interdisciplinary Material Testing and Rapid Prototyping $R \& D$ Facility" and funds from the University of Malta Research Committee.

\section{References}

[1] Miller W.S., Zhuang L., Bottema J., WitteBrood A.J., De Smet P., HASZler A., VieregGe A., Mat. Sci. Eng. A-Struct., 280 (2000), 37.

[2] European Aluminium Association, Aluminium in Cars, 9 (2008), http://www.alueurope.eu/pdf / Aluminium_in_cars_Sept2008.pdf.

[3] HiRsch J., Mater. Forum., 28 (2004), 15.

[4] Yerokhin A., Khan R.H.U., Anodising of Light Alloys, in: Dong H. (Ed.), Surface Engineering of Light Alloys: Aluminium, Magnesium and Titanium Alloys, Woodhead Publishing Ltd., Cambridge, 2010, p. 83.

[5] GhaZAli M.J., Jurnal Kejuruteraan, 18 (2006), 49.

[6] SkonecZny W., Mater. Sci.+, 46 (2010), 276.

[7] Velterop L., ATB Metallurgie, 43 (2003), 284.

[8] Runge J.M., Pomis A.J., Plat. Surf. Finish., 90 (2003), 60 
[9] Henley V.F., Anodic Oxidation of Aluminium and its Alloys, Pergamon Press, London, 1982.

[10] SADELER R., J. Mater. Sci., 41 (2006), 5803.

[11] Probert R.H., Aluminum How To: The Chromatizing, Anodizing, Hard Coating Handbook, Raleigh, NC: Tailored Text, 2005.

[12] FAng T.-H., Wang T.H., Kang S.-H., ChuAng C.-H., Curr. Appl. Phys., 9 (2009), $880-883$.

[13] Aerts T., Dimogerontakis TH., De Graeve I., Fransaer J., Terryn H., Surf. Coat. Tech., 201 (2007), 7310 .
[14] Runge J., Pomis A., 2006 EBRATS - Brazilian Surface Treatment Meeting and II Latin-American Interfinish, Sao Paulo, May 10, 2006.

[15] Critchlow G.W., Yendall K.A., Bahrani D., Quinn A., Andrews F., Int. J. Adhes. Adhes., 26 (2006), 419.
Received 2012-12-07

Accepted 2013-02-12 